Review Article Joumal of Epilepsy Research pISSN 2233-6249 / elSSN 2233-625

Received February 9, 2017

Accepted April 17, 2017

Corresponding author: Hoon-Chul Kang Department of Pediatrics, Severance Children's Hospital, Yonsei University College of Medicine, 50-1 Yonsei-ro, Seodaemoun-gu, Seoul 03722, Korea

Tel. $+82-2-2228-2050$

Fax. +82-2-393-9118

E-mail; hipo0207@yuhs.ac

\title{
Could Cannabidiol be a Treatment Option for Intractable Childhood and Adolescent Epilepsy?
}

\author{
Chung Mo Koo ${ }^{1}$, Hoon-Chul Kang ${ }^{2}$ \\ ${ }^{1}$ Department of Pediatrics, Changwon Gyeongsang National University Hospital, Changwon, Korea \\ ${ }^{2}$ Division of Pediatric Neurology, Department of Pediatrics, Severance Children's Hospital, Yonsei University \\ College of Medicine, Seoul, Korea
}

\begin{abstract}
Epilepsy is an important disease that affects brain function, particularly in those under 3 years old. Uncontrolled seizures can affect cognitive function and quality of life. For these reasons, many trials have been conducted to investigate treatments for pediatric epilepsy. Currently, many antiepileptic drugs are available for the treatment of epilepsy, but cases of intractable epilepsy continue to exist. In the past, cannabis has been tested as a potential treatment of intractable epilepsy. Since 2013,10 epilepsy centers in America have conducted research regarding the efficacy of cannabis to treat epilepsy. Cannabis has many components, including cannabidiol (CBD) and $\Delta^{9}$-tetrahydrocannabinol (THC). THC has psychoactive properties exerted through its binding of the cannabinoid receptor (CBR) whereas CBD is a CBR antagonist. The inhibition of epilepsy by CBD may therefore be caused by various mechanisms, although the detailed mechanisms of CBD actions have not yet been well defined. In most studies, trial doses of CBD were $2-5 \mathrm{mg} / \mathrm{kg} / \mathrm{day}$. Several such studies have shown that CBD does have efficacy for treatment of epilepsy. Reported adverse effects of CBD were mostly mild, including drowsiness, diarrhea, and decreased appetite. Severe adverse reactions requiring treatment, such as status epilepticus, have also been reported but it is not clear that this is related to CBD. Furthermore, many previous studies have been limited by an open-label or survey design. In future, double-blind, controlled trials are required and the use of CBD to treat other neurological problems should also be investigated. (2017;7:16-20)
\end{abstract}

Key words: Cannabis, Cannabidiol, Epilepsy

\section{Introduction}

Epilepsy impacts the brain, and is associated with cognitive dysfunction and behavior disorder, which can affect quality of life, especially during the developmental period. ${ }^{1-3}$ Early onset epilepsy, particularly in those less than 3 years old, and uncontrolled seizures are associated with poor cognitive function later in life. ${ }^{4}$

Currently, more than 20 different antiepileptic drugs (AED) exist for the treatment of epilepsy. However, 30\% of patients with epilepsy continue to have seizures. ${ }^{5-7}$ Many new medications have been approved in the past two decades, but these have not reduced the proportion of patients with intractable epilepsy. ${ }^{5}$ Recently, cannabis has attracted attention as a potential treatment of epilepsy. ${ }^{7}$ Cannabis was used to treat epilepsy in Sumeria as early as 1800 $\mathrm{BCE} .{ }^{7,8}$ In the late 19th century, English neurologists also used can- nabis for the treatment of epilepsy. ${ }^{9-11}$ More recently, some states in America have approved medical marijuana for the treatment of epilepsy. Moreover, since 2013, the effects of cannabidiol (CBD) in the treatment of epilepsy have been studied at 10 epilepsy centers using Epidiolex, a purified cannabis containing 99\% CBD and less than $0.10 \%$ tetrahydrocannabinol (THC). (GW Pharmaceuticals, Sativex, London, UK).

Cannabis contains more than 545 distinct compounds, the most abundant of which are cannabinoids. ${ }^{12}$ The most important neuroactive components of cannabis are CBD and $\Delta^{9}-\mathrm{THC}$. THC binds to G-protein-coupled cell membrane receptors, including the cannabinoid receptor type 1 (CB1R), which is found primarily in the brain, but is also present in peripheral tissues, and the cannabinoid receptor type 2 (CB2R), which is located mainly in immune and hematopoietic cells. THC binds CB1R in inhibitory gamma-aminobutyric acid-ergic 
and excitatory glutamatergic neurons. ${ }^{13,14}$ These interactions are the pathways by which cannabis exerts its psychoactive function. However, CBD is an antagonist to CB1R and CB2R. ${ }^{15}$ Some animal studies show that CB1R antagonists can reduce the threshold of seizures. ${ }^{16}$ This suggests that CBD has another mechanism by which it can control seizures. Indeed, CBD acts via multiple mechanisms (Fig. 1). ${ }^{7,14,15}$ These include effects on transient receptor potential ation channels at a low level, ${ }^{17}$ and antagonistic functions at G-protein-coupled receptor 55 , by which it can decrease presynaptic glutamate release. ${ }^{18}$ However, the mechanisms underlying the anti-

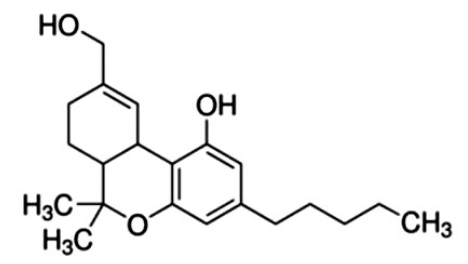

\begin{tabular}{ll}
\hline Central Nervous System target & Action \\
\hline CB1R & Antagonist \\
CB2R & Antagonist \\
GPR55 & Antagonist \\
TRPA1 & Agonist \\
TRPV1-3 & Agonist \\
TRPV4 & Agonist \\
TRPM8 & Antagonist \\
5-HT3AR & Antagonist \\
\hline
\end{tabular}

Figure 1. Cannbidiol mechanism and structure. $C B 1 R$, cannabinoid receptor type 1; CB2R, cannabinoid receptor type 2; GPR 55, G-protein-coupled receptor 55; TRPV1-4, transient receptor potential cation channel subfamily V 1-4;TRPVM8, transient receptor potential cation channel subfamily M 8; 5HT3AR, 5-hydroxytryptophan type 3A receptor. epileptic effects of CBD are not well defined.

CBD is metabolized by the liver, and it can inhibit cytochrome $P$ (CYP) 450, especially CYP 2C and CYP 3A, which aids metabolism of several AED. ${ }^{19,20}$ Therefore, if a patient with epilepsy takes CBD with an enzyme-inducing AED, such as carbamazepine or phenytoin, the $A E D$ can reduce serum CBD levels. Whereas, if the patient takes clobazam (CLB) with $C B D, C B D$ can raise the serum level of noroclobazam, the active metabolite of CLB. ${ }^{21}$ The half-life of CBD in humans is estimated to be between 18 and 32 hours. ${ }^{22}$

Here, we review recent trials that have investigated the use of CBD as a treatment for epilepsy and highlight key issues for future research.

\section{Trial dosages of CBD}

In many studies, CBD was added to a baseline AED at an initial dose of 2-5 mg/kg/day divided into two doses. CBD was up-titrated by $2-5 \mathrm{mg} / \mathrm{kg}$ once a week until intolerance or to reach a maximal dose of $25 \mathrm{mg} / \mathrm{kg} /$ day. Some studies increased the does to 50 $\mathrm{mg} / \mathrm{kg} / \mathrm{day}$, as allowed by the US Food and Drug Administration. The average of dose of CBD was 200-300 mg/day. ${ }^{7}$

\section{Clinical trials of CBD to treat epilepsy}

Some U.S. states now allow the use of cannabis for medical treatment. Many patients with epilepsy have wanted to try cannabis as a treatment even though preclinical and clinical evidence is limited. As mentioned above, several clinical studies have investigated CBD after the late 19th century. Recent studies have shown an effect of CBD in treating epilepsy (Table 1). They suggest that CBD

Table 1. Study in cannabidiol in the treatment of epilepsy

\begin{tabular}{|c|c|c|c|c|c|}
\hline Study & Age (year) & Dose & Group (n) & Results & Reference \\
\hline $\begin{array}{r}\text { Retrospective, parent } \\
\text { survey in pediatric } \\
\text { refractory epilepsy }\end{array}$ & $6-13$ & & 19 & $\begin{array}{l}\text { Complete seizer freedom: } 2 \text { patients } \\
\text { More than } 80 \% \text { reduction in seizure } \\
\text { frequency: } 8 \text { patients } \\
25-60 \% \text { seizure reduction in seizure } \\
\text { frequency: } 6 \text { patients }\end{array}$ & $\begin{array}{l}\text { Porter and } \\
\text { Jacobson }^{33}\end{array}$ \\
\hline $\begin{array}{l}\text { Retrospective, } 12-\text { month } \\
\text { trial for refractory } \\
\text { epilepsy in tuberous } \\
\text { sclerosis complex }\end{array}$ & $3-29$ & $\begin{array}{l}\text { Maximum dose of } \\
50 \mathrm{mg} / \mathrm{kg} / \text { day }\end{array}$ & 18 & $\begin{array}{l}\text { The } 50 \% \text { responder rates were } 50 \% \\
\text { after } 12 \text { months. }\end{array}$ & Hess et al. ${ }^{25}$ \\
\hline $\begin{array}{l}\text { Retrospective, open label, } \\
\text { 12- week trial in } \\
\text { refractory epilepsy }\end{array}$ & $1-22.2$ & $\begin{array}{l}\text { Maximum dose of } \\
25 \text { or } 50 \mathrm{mg} / \mathrm{kg} / \text { day }\end{array}$ & 162 & $\begin{array}{l}\text { More than } 50 \% \text { reduction in seizure } \\
\text { frequency: } 51 \text { patients } \\
\text { More than } 70 \% \text { reduction in seizure } \\
\text { frequency: } 30 \text { patients } \\
\text { More than } 90 \% \text { in seizure frequency: } \\
11 \text { patients. }\end{array}$ & Devinsky et al. ${ }^{23}$ \\
\hline
\end{tabular}


Table 2. Adverse events of cannabidiol

\begin{tabular}{|c|c|c|c|}
\hline Reference & Devinsky et al. ${ }^{23}(n=162)$ & Hess et al. ${ }^{25}(n=18)$ & Porter et al. ${ }^{33}(n=19)$ \\
\hline Drowsiness & $41(25)$ & $8(44)$ & $7(37)$ \\
\hline Behavioral problem & - & $6(31)$ & $1(16)$ \\
\hline Change in appetite & $45(28)$ & $1(5.6)$ & - \\
\hline Diarrhea & $31(19)$ & $4(22.2)$ & - \\
\hline Fatigue & $21(13)$ & - & $3(16)$ \\
\hline Convulsion & $18(11)$ & - & - \\
\hline Status epilepticus & $13(8)$ & - & - \\
\hline Lethargy & $12(7)$ & - & - \\
\hline Weight increased & $12(7)$ & - & - \\
\hline Weight decreased & $10(6)$ & - & - \\
\hline Ataxia & $9(6)$ & $5(27.8)$ & - \\
\hline Increased self-stimulation & - & $1(5.6)$ & - \\
\hline
\end{tabular}

Values are presented as number (\%).

can reduce the frequency of seizures, reducing monthly frequency in most types of seizures. Many studies have included a significant number of patients with Dravet syndrome (DS) and Lennox-Gastaut syndrome (LGS), and have observed reduced seizures as an effect of CBD.

Between 2014 and 2015, Devinsky et al. ${ }^{23}$ conducted a prospective, open-label, and expanded trial at 11 epilepsy centers in America. They enrolled 214 patients in the study, of which $137(64 \%)$ were included in an efficacy analysis group and 162 (76\%) were included in a safety analysis group. They observed enrolled patients for 12 weeks. This study reported a decrease of $34.6 \%$ in the median change of total seizures after 12 weeks' treatment with CBD. It also showed a decrease of $55 \%$ in focal seizures and $54.3 \%$ in atonic seizures. They observed a decrease of $36.5 \%$ and $16 \%$ in tonic seizures and tonic-clonic seizures, respectively. Overall, the median monthly frequency of motor seizures decreased by $47 \%$.

This open-label trial enrolled a large portion of patients with DS $(n=32)$ or LGS $(n=30)$. In the case of DS, 16 patients $(50 \%)$ showed a reduction in motor seizures of more than $50 \%$, and one patient (3\%) was free from motor seizures during the 12 weeks of treatment. They reported a median change of $-69.2 \%$ in monthly tonic seizures $(n=6)$ and a median change of $-46.7 \%$ in monthly tonic-clonic seizures $(n=29)$. Non-motor-focal seizures $(n=10)$ were observed to show a median reduction of $83.3 \%$. Among patients with $\operatorname{LGS}(n=30), 11(37 \%)$ showed a decrease in seizures of more than $50 \%$, but none were seizure free during treatment. The percentage change in monthly seizures for all seizure types was $-35.5 \%$. The median change for atonic seizures $(n=14)$ was $-68.8 \%$. In patients with tonic seizures $(n=21)$, there was a median reduction of $44.0 \%$. However, there was no change in tonic-clonic seizures $(n=16)$.

Infantile spasm (IS) is another epileptic encephalopathy in need of an effective treatment. In 2014, Hussain et al. conducted a survey about CBD exposure, ${ }^{24}$ which included patients with IS and LGS. Although this study had several limitations, it supported the possibility that CBD could effectively treat IS and LGS.

Hess et al. studied the efficacy of CBD for refractory epilepsy in tuberous sclerosis complex (TSC). ${ }^{25}$ They enrolled 18 patients with epilepsy and TSC between 2014 and 2015, and treated them for 6-12 months with CBD. In total, 14 patients (77\%) had a mutation, half of whom had a TSC1 mutation and the other half of whom had a TSC2 mutation. At baseline, $72 \%$ of patients had shown one or more type of seizure. ${ }^{25}$ In this study, the median total weekly seizure frequency reduced by $32 \%$ after 2 months and $55 \%$ after 6 months. At study completion after 12 months of treatment, the seizure frequency was decreased to $63 \%$. Regarding the CBD effect on each type of seizure, epileptic spasms and atonic seizures showed the greatest response. The responder rate of tonic-clonic seizures was $66.7 \%$ after 3 months' treatment. The rates of complex partial seizures and complex partial seizures with secondary generalization were $53.8 \%$ and $50 \%$ after 3 months' treatment, respectively. This study was limited by a small sample size, as well as an uncontrolled and unblinded design, but suggests a potential effect of CBD in TSC.

\section{Reported adverse effects}

In previous studies, the most common adverse effect of $C B D$ was drowsiness. Most reported adverse effects were mild, for example diarrhea, fatigue, and decreased appetite. Nonetheless, some more serious effects, including status epilepticus, have been reported. 
Devinsky et al. reported adverse events in 128 (79\%) of 162 recruited patients. ${ }^{23}$ Most of these adverse effects were mild or moderate, and transient. Critical adverse effects considered to be caused by CBD were observed in 20 patients (12\%). ${ }^{23}$ The most common adverse event was somnolence, while status epilepticus was the most severe and required urgent treatment (Table 2). It is interesting that CBD had this severe adverse effect, status epilepticus, because animal studies have not shown any proconvulsant effects of $C B D ., 26$ Devinsky et al. also reported thrombocytopenia in 5 patients (3\%), but there were no clinical changes in white or red blood cells. CBD also did not affect renal function. Ten patients (6\%) showed elevated liver enzymes and this elevation was significant in one patient $(<1 \%)$. The authors did not find any relationship between status epilepticus and reduced doses of AED or CBD. Four patients stopped $\mathrm{CBD}$ treatment before the end of 12 weeks because of a worsening of seizures or poor efficacy.

\section{Discussion}

Most studies suggest anticonvulsant effects of $\mathrm{CBD}$, and consider most adverse effects to be mild. As such, we might expect CBD to show efficacy and safety in the treatment of epilepsy. However, there is potential placebo effects biasing these results. In a study by Purcarin et al. ${ }^{27}$ the placebo effect rate for the reduction of median seizure frequency was $10 \%$ and the placebo effect was $19 \%$ in cases in which seizure frequency decreased by $50 \%$ or more. According to the study of Zaccara et al. ${ }^{28}$ greater placebo effects were observed in pediatric patients with epilepsy treated with CBD than were in adults. A placebo effect is a concern for many studies because of the media attention attracted by CBD, which is likely to interest parents of children with epilepsy. In most studies, seizure frequency was used as an indicator of efficacy. However, to increase the accuracy of proposed effects, data from clinical tests, such as electroencephalograms, are necessary.

Due to the absence of a control group in previous studies, the adverse reactions to CBD may also have resulted from a bias. For example, many studies reported gastrointestinal problems, such as diarrhea and decreased appetite. However, CBD is usually in the form of oil, which may have precipitated these effects. Therefore, the adverse effects should be reviewed. Furthermore, other adverse effects, such as drowsiness, thrombocytopenia, and elevated liver enzymes, also require more research to exclude the potential influence of AEDS, such as clobazam and valproate. Status epilepticus, the most com- mon severe adverse effect, was not clearly related to CBD because a large proportion of the enrolled patients had refractory epilepsy syndromes, such as LGS, DS and IS.

CBD not only has potential therapeutic effects on epilepsy, but may also be effective in treating various other neurological problems. ${ }^{9}$ In one study, CBD showed antioxidant, anti-inflammatory, neuroproliferative, and remyelinating effects. ${ }^{29}$ While cannabinoid receptor agonists can reduce hypoxic-ischemic brain injury, ${ }_{1}^{29}$ lasting overactivation of CB1R can have potential disadvantages. However, CBD does not have agonist effects on CB1R and so can be an alternative treatment. ${ }^{30}$ Furthermore, $C B 2 R$ is one of the primary receptors affected by $C B D$ action in the immature brain. ${ }^{30,31}$ In animal studies, CBD has been shown to reduce injury to neurons and astrocytes due to hypoxia. ${ }^{31,32}$ This suggests that neonatal hypoxic ischemic encephalopathy could be treated with CBD. This should be a focus of future studies.

One of the most significant limitations of previous studies is their open-label, uncontrolled design and the use surveys. Future research should use blinded, randomized, controlled trials to confirm the efficacy and adverse effects without bias. Currently, many studies are limited to epilepsy, mainly enrolling patients with LGS and DS. Further studies of other epilepsy syndromes are also needed in future.

It must be borne in mind that CBD is still illegal in many contexts. However, it has the potential to treat various neurological problems, including epilepsy. We should seek to clarify the mechanisms underlying the antiepileptic effects of CBD and its efficacy for other neurologic problems. Furthermore, drug-drug interactions with AEDs require further research. Previous studies have determined exact therapeutic drug levels of CBD in the blood. Therefore, this also needs to be clarified to enable effective treatment of epilepsy with CBD.

\section{Acknowledgements}

This research was supported by a grant of the Korea Health Technology R\&D Project through the Korea Health Industry Development Institute (KHIDI), funded by the Ministry of Health \& Welfare, Republic of Korea (grant number : HI15C1601).

\section{References}

1. Devinsky O, Vickrey BG, Cramer J, et al. Development of the quality of life in epilepsy inventory. Epilepsia 1995;36:1089-104. 
2. Donner EJ. Opportunity gained, opportunity lost: treating pharmacoresistant epilepsy in children. Epilepsia 2013;54 Suppl 2:16-8.

3. Cilio MR, Thiele EA, Devinsky 0. The case for assessing cannabidiol in epilepsy. Epilepsia 2014;55:787-90.

4. Berg AT, Zelko FA, Levy SR, Testa FM. Age at onset of epilepsy, pharmacoresistance, and cognitive outcomes: a prospective cohort study. Neurology 2012;79:1384-91.

5. Brodie MJ, Barry SJ, Bamagous GA, Norrie JD, Kwan P. Patterns of treatment response in newly diagnosed epilepsy. Neurology 2012;78:1548-54.

6. Kwan P, Brodie MJ. Early identification of refractory epilepsy. $N$ Engl J Med 2000;342:314-9.

7. Longo DL, Friedman D, Devinsky O. Cannabinoids in the treatment of epilepsy. N Eng/ J Med 2015;373:1048-58.

8. Schultes RE. Man and marihuana. Nat Hist 1973;82:59-68.

9. Devinsky O, Cilio MR, Cross $H$, et al. Cannabidiol: pharmacology and potential therapeutic role in epilepsy and other neuropsychiatric disorders. Epilepsia 2014;55:791-802.

10. Reynolds JR. Epilepsy: its symptoms, treatment, and relation to other chronic, convulsive diseases. Am J Psychiatry 1862;19:198-209.

11. Gowers WR. Epilepsy and other chronic convulsive disorders, their causes, symptoms and treatment. London: Churchill 1881, p. 223.

12. ElSohly M, Gul W. Constituents of cannabis sativa. Handbook of Cannabis. Oxford: Oxford University Press;2014.

13. Lutz B. On-demand activation of the endocannabinoid system in the control of neuronal excitability and epileptiform seizures. Biochem Pharmacol 2004;68:1691-8.

14. Pertwee RG, Cascio MG. Known pharmacological actions of Delta-9-tetrahydrocannabinol and of four other chemical constituents of cannabis that activate cannabinoid receptors. In: Handbook of Cannabis. Oxford: Oxford University Press; 2014.

15. Cascio MG, Pertwee RG. Known pharmacological actions of nine nonpsychotropic phytocannabinoids. In: Handbook of Cannabis. Oxford: Oxford University Press; 2014.

16. Wallace MJ, Blair RE, Falenski KW, Martin BR, DeLorenzo RJ. The endogenous cannabinoid system regulates seizure frequency and duration in a model of temporal lobe epilepsy. I Pharmacol Exp Ther 2003;307:129-37.

17. De Petrocellis L, Ligresti $A$, Moriello AS, et al. Effects of cannabinoids and cannabinoid-enriched Cannabis extracts on TRP channels and endocannabinoid metabolic enzymes. Br J Pharmaco/.2011;163:1479-94.

18. Sylantyev S, Jensen TP, Ross RA, Rusakov DA. Cannabinoid-and lysophosphatidylinositol-sensitive receptor GPR55 boosts neurotransmitter release at central synapses. Proc Natl Acad Sci USA 2013;110:5193-8.

19. Stout SM, Cimino NM. Exogenous cannabinoids as substrates, in- hibitors, and inducers of human drug metabolizing enzymes: a systematic review. Drug Metab Rev 2014;46:86-95.

20. Patsalos PN, Perucca E. Clinically important drug interactions in epilepsy: general features and interactions between antiepileptic drugs. Lancet Neurol 2003;2:347-56.

21. Geffrey AL, Pollack SF, Bruno PL, Thiele EA. Drug-drug interaction between clobazam and cannabidiol in children with refractory epilepsy. Epilepsia 2015;56:1246-51.

22. Hawksworth G, McArdle K. Metabolism and pharmacokinetics of cannabinoids. Pharmaceutical Press, London, UK; 2004.

23. Devinsky 0 , Marsh $E$, Friedman $D$, et al. Cannabidiol in patients with treatment-resistant epilepsy: an open-label interventional trial. Lancet Neurol 2016;15:270-8.

24. Hussain SA, Zhou R, Jacobson C, et al. Perceived efficacy of cannabidiol-enriched cannabis extracts for treatment of pediatric epilepsy: A potential role for infantile spasms and Lennox-Gastaut syndrome. Epilepsy Behav 2015;47:138-41.

25. Hess EJ, Moody KA, Geffrey AL, et al. Cannabidiol as a new treatment for drug-resistant epilepsy in tuberous sclerosis complex. Epilepsia 2016;57:1617-24.

26. Hill AJ, Hill TD, Whalley B. The development of cannabinoid based therapies for epilepsy. Endocannabinoids: molecular, pharmacological, behavioral and clinical features bentham science publishers, Oak Park, IL 2013;164-204.

27. Purcarin $\mathrm{G}, \mathrm{Ng}$ YT. Experience in the use of clobazam in the treatment of Lennox-Gastaut syndrome. Ther Adv Neurol Disord 2014;7:169-76.

28. Zaccara G, Giovannelli F, Schmidt D. Placebo and nocebo responses in drug trials of epilepsy. Epilepsy Behav 2015;43:128-34.

29. Martinez-Orgado J, Fernandez-Lopez D. The seek of neuroprotection: introducing cannabinoids. Recent Pat CNS Drug Discov 2007;2:131-39.

30. Castillo A, Tolón MR, Fernández-Ruiz J, Romero J, Martinez-Orgado J. The neuroprotective effect of cannabidiol in an in vitro model of newborn hypoxic-ischemic brain damage in mice is mediated by $\mathrm{CB}(2)$ and adenosine receptors. Neurobiol Dis 2010;37:434-40.

31. Pazos MR, Mohammed N, Lafuente $H$, et al. Mechanisms of cannabidiol neuroprotection in hypoxic-ischemic newborn pigs: role of 5HT(1A) and CB2 receptors. Neuropharmacology 2013;71:282-91.

32. Lafuente $H$, Alvarez FJ, Pazos MR, et al. Cannabidiol reduces brain damage and improves functional recovery after acute hypoxia-ischemia in newborn pigs. Pediatr Res 2011;70:272-7.

33. Porter $B E$, Jacobson C. Report of a parent survey of cannabidiol-enriched cannabis use in pediatric treatment-resistant epilepsy. Epilepsy Behav 2013;29:574-7. 